\title{
Pesquisa participante baseada na comunidade: fundamentos, requisitos e desafios ao pesquisador
}

Community based participatory research: foundations, requirements and challenges to researcher

Investigación participante basada en la comunidad: fundamentos, requisitos y desafíos para el investigador

\section{Cristiane José Borges ${ }^{\mathrm{I}}$, Denize Bouttelet Munari ${ }^{\mathrm{II}}$, Veridiana Carvalho Bianco ${ }^{\mathrm{III}}$, Paula Cândida da Silva Diasiv, Marcelo Medeirosv, Jeanne-Marie Rodrigues Stacciarini ${ }^{\mathrm{II}}$}

\begin{abstract}
Resumo: Objetivo: descrever fundamentos teórico-filosóficos e possíveis aplicações da Pesquisa Participante Baseada na Comunidade. Método: reflexão teórica focada no potencial transformador dessa abordagem no âmbito da Saúde Pública. Resultados: nessa modalidade de pesquisa, parceiros da comunidade são valorizados em todas as fases do processo investigativo, auxiliando no delineamento de intervenções que atendam suas necessidades. Além disso, são criados espaços, instrumentos de produção e divulgação de ideias, práticas e alternativas de cuidado, a fim de facilitar a transposição de barreiras de linguagem e comunicação entre profissionais dos serviços, pesquisadores e usuários do sistema de saúde. No contexto da Pesquisa Participante Baseada na Comunidade o gerenciamento diário do cuidado fundamenta-se no diálogo cultural e do fazer compartilhado, o que desenvolve confiança mútua e sustentabilidade do processo de interação pesquisador - comunidade. Considerações finais: a Pesquisa Participante Baseada na Comunidade é importante abordagem metodológica para compreender a complexidade humana e avanços da enfermagem comunitária.
\end{abstract}

Descritores: Pesquisa participativa baseada na comunidade; Enfermagem em saúde comunitária; Pesquisa em enfermagem; Promoção da saúde.

\footnotetext{
${ }^{\text {I }}$ Enfermeira. Doutora em Enfermagem. Universidade Federal de Goiás. Jatai, Goiás, Brasil. E-mail: cristianejose@yahoo.com.br; ORCID: https://orcid.org/0000-0002-5846-0750

II Enfermeira. Doutora em Enfermagem. Universidade Federal de Goiás. Goiânia, Goiás, Brasil. E-mail: boutteletmunari@gmail.com; ORCID: https://orcid.org/0000-0002-2225-770X

III Enfermeira. Graduada pelo Curso de Graduação em Enfermagem da Universidade Federal de Goiás. Jataí, Goiás, Brasil. E-mail: veridianacbianco@gmail.com ORCID: https://orcid.org/0000-0002-0291-9518

IV Enfermeira. Doutoranda FEN/UFG, Mestre em Atenção à Saúde. Pontifícia Universidade Católica de Goiás, Goiânia, Goiás, Brasil. Email:paulacandidadias@gmail.com; ORCID:http//orcid.org/0000-0002-87610551

v Enfermeiro. Doutor em Enfermagem. Faculdade de Enfermagem da Universidade Federal de Goiás, Goiânia, Goiás, Brasil..Email:Marcelo@ufg.br; ORCID: https://orcid.org/0000-0001-6979-321

${ }^{\text {VI }}$ Nurse. RN, PhD, FAAN. Associate Professor. Assistant Dean for Diversity, Inclusion and Global Affairs. College of Nursing - University of Florida Gainesville, F. Email: jeannems@ufl.edu ORCID: https://orcid.org/0000-0003-0261-8413
} 
Pesquisa participante baseada na comunidade: fundamentos, requisitos e desafios ao pesquisador I 2

\begin{abstract}
Objective: To describe theoretic and philosophic foundations and possible applications of Community Based Participatory Research. Method: theoretical reflection focused on this approach's transformative potential in Public Health scope. Results: in this research modality, community partners are valued at all stages of the investigative process, helping to design interventions that meet their needs. In addition, spaces and production instruments are created, and ideas, practices and care alternatives are disseminated, in order to facilitate the transposition of language and communication barriers between service professionals, researchers and health system users. In the Community Based Participatory Research context, daily care management is based on cultural dialogue and sharing of chores, which develops mutual trust and sustainability of the researcher-community interaction process. Final Considerations: Community Based Participatory Research is an important methodological approach to understand the human complexity and advances in community nursing.

Descriptors: Community based participatory research; Community health nursing; Nursing research; Health promotion.
\end{abstract}

Resumen: Objetivo: Describir fundamentos teóricos-filosóficos y posibles aplicaciones de la investigación participante basada en la comunidad. Método: reflexión teórica con foco en el potencial transformador del abordaje en el ámbito de la salud pública. Resultados: En esa modalidad de investigación, compañeros de la comunidad son valorizados en todas las fases de investigación, auxiliando en el delineamiento de intervenciones que atiendan sus necesidades. Son creados espacios, instrumentos de producción y divulgación de ideas, prácticas y alternativas de cuidado, así facilitar y transponer barreras de lenguaje y comunicación entre profesionales, investigadores y usuarios del sistema de salud. La investigación participante basada en la comunidad, el gerenciamiento del cuidado se fundamenta en el dialogo cultural y realizar en conjunto, lo que desenvuelve confianza mutua y sustentabilidad de interacción investigador-comunidad. Consideraciones finales: La pesquisa participante basada en la comunidad es importante el abordaje metodológico para comprender la complexidad humana y avances de la enfermería comunitaria.

Descriptores: Investigación participativa basada en la comunidad; Enfermería en salud comunitaria; Investigación en enfermería; Promoción de la salud.

\title{
Introdução
}

A enfermagem brasileira tem crescido como ciência e profissão por desenvolver significativo conhecimento científico, bem como, com o crescimento da sua massa crítica, sobretudo, viabilizada pela expansão dos programas de pós-graduação. Esses movimentos têm fortalecido a produção de novas pesquisas e conferido visibilidade à profissão como ciência. ${ }^{1}$

No entanto, estudo nacional ${ }^{2}$ menciona desafios aos pesquisadores da enfermagem para a utilização de metodologias mais robustas, capazes de produzir maior impacto e conhecimento passível de ser incorporado imediatamente na prática, e/ou trazer inovações em prol do cuidado oferecido. $\mathrm{Na}$ área da saúde, de modo geral, também a incorporação de inovações que produzam 
3 | Borges CJ, Munari DB, Bianco VC, Dias PCS, Medeiros M, Stacciarini JMR

transformações das práticas e que se baseiem nos fundamentos do sistema de saúde brasileiro constituem grande desafio, o que incita a comunidade científica a buscar resultados mais efetivos. $^{2}$

Tal cenário explicita, entre outros aspectos, a necessidade de se criar espaços para o desenvolvimento de tecnologias sociais, as quais permitam aos usuários dos serviços de saúde maior protagonismo. Também favorece a definição de prioridades em pesquisa, auxiliando na identificação de experiências exitosas de inovação na atenção em saúde e valorizando as iniciativas existentes..$^{2-3}$ Essas tendências indicam aos pesquisadores que as investigações precisam, além de preencher as lacunas do conhecimento, serem pertinentes às necessidades reais dos usuários dos serviços de saúde. Esse processo pode gerar mais impacto na melhoria da qualidade de vida dessas pessoas e torná-las mais ativas. Dessa forma, será possível construir uma sociedade mais democrática e desenvolver práticas socioculturalmente apropriadas, que facilitem a transferência de conhecimento científico para a prática clínica e assistencial.-3

Nesta perspectiva, estudo ${ }^{3}$ assinala que o profissional enfermeiro, ao planejar um estudo na comunidade, precisa ter clareza do que espera alcançar como produto, sobretudo, o seu impacto para a comunidade parceira da pesquisa. Deve também se certificar de que os resultados das pesquisas serão capazes de contribuir para o aprimoramento de ações e serviços de saúde provenientes dos setores público e privado; influenciar as políticas públicas de saúde; melhorar a saúde, o bem-estar e a qualidade de vida humana; e minimizar as disparidades no acesso aos cuidados em saúde. ${ }^{3}$ Tais recomendações se alinham aos princípios do sistema de saúde brasileiro e da defesa dos direitos democráticos à saúde.

Nesse sentido, os fundamentos da Pesquisa Acoplada à Comunidade (PAC) apresentam elementos que valorizam e reconhecem a importância de todos os envolvidos na produção do conhecimento científico, ou seja, comunidade, pesquisadores e instituições, o que demonstra se 
Pesquisa participante baseada na comunidade: fundamentos, requisitos e desafios ao pesquisador I 4

tratar uma abordagem que privilegia ações democráticas e de engajamento social no processo investigativo.

Embora exista uma grande diversidade de tipologias para o delineamento de uma PAC, ${ }^{3}$ destaca-se, no presente artigo, a Pesquisa Participante Baseada na Comunidade (PPBC). Considerada uma abordagem transformadora no âmbito da saúde pública, deve ser aplicada por investigadores que se preocupam com os problemas e as desigualdades sociais de determinada comunidade, em especial, as minoritárias ou excluídas. Além disso, tem como escopo a redução das lacunas existentes entre o conhecimento produzido por meio da pesquisa convencional e as necessidades da comunidade. ${ }^{4}$

Buscas na literatura para identificar estudos conduzidos com uso da abordagem da PPBC mostrou ampla utilização no contexto internacional, em particular, no Canadá e nos Estados Unidos da América. ${ }^{3,5-6}$ Esses estudos sinalizam que o caminho mais efetivo para melhorar a saúde da comunidade é envolvê-la no processo de investigação, de modo que suas carências possam ser atendidas com mais resolutividade e efetividade. ${ }^{5-6}$ Indicam também que essas pesquisas devem ser culturalmente sensíveis à população, ${ }^{3}$ com vistas a reduzir as desigualdades socioculturais e políticas.

A proposta deste estudo, baseia-se nos princípios da PPBC, sobretudo no que se refere, à produção de conhecimento em parceria com a comunidade e à necessidade de envolver os usuários dos serviços de saúde de forma mais ativa nos processos de mudanças nas práticas em saúde. $^{2}$

Nessa perspectiva, este artigo se constitui em uma reflexão teórica focada no potencial transformador desta modalidade de pesquisa no âmbito da Saúde Pública, partindo da seguinte questão norteadora: qual o potencial do uso da Pesquisa Participante Baseada na Comunidade no desenvolvimento de pesquisas em saúde coletiva? 
5 | Borges CJ, Munari DB, Bianco VC, Dias PCS, Medeiros M, Stacciarini JMR

Assim, tendo em vista que o uso da PPBC é incipiente na enfermagem brasileira, delineou-se como objetivo do presente artigo descrever fundamentos teórico-filosóficos e possíveis aplicações da Pesquisa Participante Baseada na Comunidade.

\section{Conceitos e bases teórico-filosóficas da Pesquisa Participante Baseada na Comunidade}

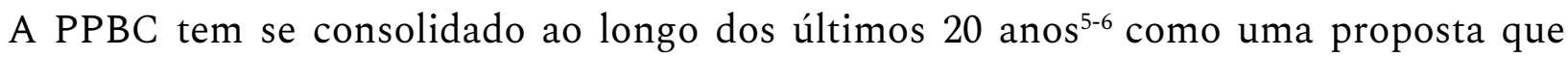
integra dois referenciais teóricos. O primeiro baseia-se no diálogo dos fundamentos da Pesquisa Ação (PA) de Kurt Lewin, ${ }^{4}$ sustentada na proposição de uma ação ou a resolução de um problema coletivo, em que pesquisadores e participantes estão envolvidos de modo cooperativo e participativo com o intuito de melhorar a prática assistencial em saúde., ${ }^{4,6} \mathrm{O}$ segundo, fundamenta-se nos pressupostos da educação libertadora de Paulo Freire ${ }^{7-8}$ como um caminho para minimizar as desigualdades sociais existentes na comunidade.,

A influência Freiriana na PPBC projeta as ideias deste grande pensador brasileiro de que o indivíduo precisa ter compreensão crítica, global e significativa do contexto em que vive. Tanto na PA quanto na educação libertadora, a participação ativa de todos os sujeitos envolvidos é condição para a construção de mudanças e efetivas transformações sociais..$^{7-8}$

Os primeiros registros da criação e elaboração dos conceitos originais da PPBC surgiram no Canadá em 1995, nos Estados Unidos, em 1998. Por definição, é considerada uma abordagem de pesquisa cuja finalidade é investigar e, simultaneamente, promover mudança em prol da comunidade, mediante a colaboração ativa das pessoas, em especial, de grupos minoritários e afetados pelo mesmo problema. ${ }^{10-11}$

Em 2004, após análise dos diversos conceitos acerca da PPBC desenvolvidos no Canadá e nos Estados Unidos, a Agência Americana de Pesquisa e Qualidade em Saúde, responsável pelo desenvolvimento de pesquisa e qualidade do cuidado à saúde, estabeleceu defini-la como um tipo de abordagem colaborativa capaz de agregar, em sua estrutura formal, estratégias para 
Pesquisa participante baseada na comunidade: fundamentos, requisitos e desafios ao pesquisador I 6

que ocorra a participação ativa da comunidade pesquisada. Essa participação conjunta deve acontecer em todas as etapas do estudo, desde o delineamento do problema investigado até a análise e divulgação dos resultados. ${ }^{12}$

Ademais, tal abordagem propicia a integração entre membros da comunidade, os pesquisadores e as instituições envolvidas no processo de investigação. Seu maior propósito reside melhorar a saúde e o bem-estar dos indivíduos, mediante a construção de decisões compartilhadas, que vislumbrem significativas transformações no contexto social em que vivem os participantes em estudo. ${ }^{4}$

Nessa abordagem, também se contempla uma pluralidade de saberes, o que oportuniza a coaprendizagem, sendo esta pautada na responsabilidade e no respeito mútuo entre todos os parceiros envolvidos no método de investigação científica. ${ }^{13}$

O objeto de interesse da comunidade é elemento-chave para aproximar e envolver outros atores, como profissionais de saúde, educação, segurança pública, alunos de graduação e pós-graduação, religiosos, entre outros. A depender desse envolvimento e da proposta de transformação definida pela comunidade é possível estabelecer parcerias que integrem os interesses acadêmicos com os serviços comunitários.

Na PPBC, a compreensão de um determinado fenômeno somente ocorre se houver efetiva contribuição de uma comunidade, para a qual ele faça sentido e reflita uma necessidade real de mudança. Assim, o conhecimento adquirido é integrado por meio da implementação de intervenções imediatas e efetivas, apoiado nas políticas de saúde e qualidade de vida dos membros da comunidade..$^{3,5,13}$ Nesta perspectiva, a abordagem metodológica da PPBC permite combinar métodos de pesquisa e estratégias que incluam a comunidade no processo investigatório. Tem, finalmente, como finalidade minimizar a lacuna existente entre o conhecimento produzido por meio da pesquisa tradicional e a aplicação dos seus resultados para fundamentar a implantação e/ou implementação de intervenções e políticas. ${ }^{8,13}$ 
Por permitir a integração de variados métodos para o alcance dos objetivos preestabelecidos na condução da investigação, a depender dos interesses da comunidade, a PPBC é reconhecida como uma abordagem ampla de pesquisa e não simplesmente como um método hermético em si..$^{3-5,11}$ Assim, tem sido largamente utilizada em investigações no campo da saúde direcionadas para o desenvolvimento de intervenções de promoção da saúde, do meio ambiente e no desenvolvimento de políticas públicas. ${ }^{8,10,13-15}$

Pode também ser aplicada em diferentes cenários, inclusive por pesquisadores de mais de uma área do conhecimento. $\cdot^{3-4,6,8-9,12} \mathrm{Na}$ prática, essa abordagem metodológica tem sido instrumental no processo de identificar e minimizar os determinantes sociais de saúde. É reconhecida por pesquisadores, profissionais, estudantes e membros da comunidade como uma estratégia eficaz para a resolutividade dos problemas que envolvem este setor, os quais são complexos e exigem soluções multifacetadas nas diversas esferas: política, social e biomédica. ${ }^{4}$

Pesquisas desenvolvidas com base nessa abordagem têm comprovado o seu potencial no desenvolvimento de comunidades e no empoderamento de pessoas que, as quais tiveram oportunidade de assumir o protagonismo social e têm sido capazes de promover inúmeros benefícios coletivos. ${ }^{3,5,8,10,13-15}$

Além das bases conceituais que fundamentam os princípios de estudos conduzidos pela PPBC é essencial aos pesquisadores que se propõem a realizar estudos com essa abordagem conhecer requisitos para se empreender investigações dessa natureza. O conhecimento da complexidade da vida comunitária, o respeito às diferenças e aos pontos de vista de cada participante é apenas uma das facetas que se precisa considerar no planejamento da investigação. A seguir detalhamos esses, entre outros, requisitos para garantia do rigor na condução de pesquisas que utilizam a PPCB. 
Pesquisa participante baseada na comunidade: fundamentos, requisitos e desafios ao pesquisador 18

Requisitos essenciais para o desenvolvimento da Pesquisa Participante Baseada na Comunidade

A PPBC não é concebida como um método hermético em si, mas como uma abordagem/orientação de pesquisa que influencia diretamente a maneira como os pesquisadores e participantes se relacionam, independentede suas origens. ${ }^{4,8-9,11} \mathrm{~A}$ relação entre pesquisadores e pesquisados é de parceria estreita e horizontal, baseada no respeito e valorização das diversidades culturais..$^{4,8-9}$ Trata-se de um processo de investigação realizado no mesmo contexto em que são identificadas as necessidades e demandas da comunidade., ${ }^{4,9,11}$

$\mathrm{Na}$ condução da PPBC é indispensável se manter diálogo ativo e respeitoso entre os parceiros representantes da academia e da comunidade, a fim de favorecer o empoderamento de todos os indivíduos. A identificação de conhecimentos, competências e habilidades de cada um dos parceiros da investigação é fundamental para se utilizar todo o potencial da comunidade, inclusive de seus saberes e práticas. ${ }^{4,9}$

Nesse contexto, a comunicação confiável e o poder compartilhado são princípios básicos para a tomada de decisão igualitária em todas as fases da PPBC, desde a construção das questões de pesquisa, até a coleta, análise, interpretação dos dados e divulgação dos resultados. ${ }^{4-}$ ${ }^{5,16}$ Esse processo requer alto nível de confiança entre todos os parceiros envolvidos durante a investigação. ${ }^{4-5,12,16}$ Além disso, o pesquisador deve estar ciente de que este tipo de abordagem se diferencia de uma pesquisa tradicional no que se refere ao papel da comunidade, especialmente devido ao processo comunicacional de respeito as diferenças e estímulo a participação ativa dos parceiros. ${ }^{16}$ Nesse caso, trabalha-se com e em comunidade, sendo a tríade Participação Baseada na Comunidade $(\mathrm{PBC})$ o foco principal. ${ }^{4,13} \mathrm{~A}$ comunidade é compreendida como uma entidade psicossociocultural, sendo que a participação ativa dos seus membros med,8-9 $^{3-c o r r e ~ m e d i a n t e ~ a ~}$ criação de um Comitê de Assessoria Comunitária (CAC). ${ }^{3-5}$ 
Na PPBC, o CAC representa o principal elemento da condução para participação ativa e do empoderamento dos parceiros comunitários em todo o processo de investigação científica. ${ }^{4}$ A composição e o número de participantes neste tipo de comitê variam de acordo com o contexto no qual o estudo está sendo realizado, sem que regras sejam preestabelecidas. ${ }^{6,11}$ Geralmente, é composto por indivíduos com envolvimento e interesse na resolução de problemas (exemplo: membros da comunidade, profissionais de saúde, membros de grupos religiosos ou de apoio social, entre outros), que possuem características heterogêneas, interesses diversos com relação ao problema estudado e diferentes níveis educacionais, sociais, crenças, valores e conhecimentos. ${ }^{11}$

Esta diversidade de olhares do CAC possibilita que a PPBC exerça impacto direto no cenário estudado, o que reverte em melhorias para a comunidade, tais como fortalecimento de parcerias e da potencialização dos recursos comunitários existentes. Assim, as propostas para solucionar ou minimizar os problemas detectados são mais eficientes e o desenvolvimento da pesquisa e a transformação da comunidade afetada acontecem simultaneamente. ${ }^{4}$

A PPBC baseia-se em nove princípios, conforme ilustra o quadro 1:

1. O reconhecimento da comunidade como unidade de identidade;

2. A pesquisa é concebida de acordo com a capacidade e os recursos da própria comunidade;

3. A pesquisa realiza-se por meio de parceria colaborativa e equitativa entre os pesquisadores e a comunidade em todas as fases da investigação, mediante capacitação e empoderamento de todos os parceiros de maneira a reduzir as desigualdades sociais;

4. A pesquisa deve promover a coaprendizagem e capacitação de todos os parceiros;

5. Integrar o conhecimento e ações em prol de benefícios mútuos para todos os parceiros;

6. Focar na relevância local dos problemas de saúde pública por meio de perspectivas ecológicas que englobem os múltiplos determinantes de saúde;

7. A pesquisa se desenvolve mediante um processo interativo e cíclico;

8. Os resultados obtidos devem ser divulgados a todos os parceiros, os quais devem ajudar na disseminação dos dados;

9. Requer envolvimento de longo prazo e comprometimento com a sustentabilidade.

Quadro 1 - Princípios essenciais para o desenvolvimento da PPBC 4:6-9 
Pesquisa participante baseada na comunidade: fundamentos, requisitos e desafios ao pesquisador | 10

Na prática, compete ao pesquisador assegurar, por meio da formação do CAC e das ações desenvolvidas por seus integrantes, que esses princípios sejam contemplados. Todavia, as estratégias para garantir a observância desses princípios serão construídas no decorrer do processo investigativo, mediante análise e reflexão coletiva a respeito da realidade da comunidade pesquisada.

Relevante destacar um dos fundamentos que orientam a PPBC: associar a valorização do conhecimento compartilhado entre pesquisador e comunidade, a maximização da relevância dos resultados e a garantia do rigor metodológico, exigida em qualquer tipo de estudo científico.-6 $\mathrm{O}$ rigor da PPBC para com a produção do conhecimento acoplado aintervenções comunitárias, provavelmente não seria possível com a aplicação de outros métodos. Além disso, na definição coletiva do(s) problema(s) de pesquisa, assim como no delineamento dos objetivos e do trajeto metodológico, é possível obter dados de natureza qualitativa e quantitativa.-6,9,11,16

A complexidade de uma investigação conduzida em consonância com os pressupostos da PPBC impõem aos pesquisadores, que devem ser cuidadosamente pensados na fase do planejamento da investigação, embora muitos deles surjam apenas no decorrer do processo investigativo. No tópico a seguir serão destacados alguns desafios descritos na literatura e também observados com frequência durante a utilização dessa abordagem.

\section{Desafios para o pesquisador e a comunidade envolvidos na pesquisa participante baseada na comunidade}

Apesar da PPBC estar consolidada no meio científico internacional há algumas décadas, os pesquisadores ainda se deparam com dificuldades ao conduzi-la. ${ }^{12,17}$ Evidências indicam que uma das limitações na condução da PPBC envolve a necessidade de assegurar o envolvimento equitativo dos parceiros e o equilíbrio entre método de investigação e as intervenções relevantes

à comunidade. ${ }^{3,6,16}$ Para se garantir esse equilíbrio e os princípios da abordagem, é fundamental 
que o pesquisador tenha uma visão ampliada do processo investigativo, pois assim ele e sua equipe poderão identificar mais facilmente eventuais tentativas de se recorrer às origens do pensamento positivista na resolução das demandas da pesquisa, comuns à maioria dos pesquisadores.

Dessa forma, quem utiliza a abordagem da PPBC precisa estar ciente de investigar um problema sob a perspectiva de um novo paradigma. Isso requer uma significativa inserção do pesquisador na comunidade, além de sensibilidade e resiliência para aceitar visões diferentes das já consolidadas na cultura da investigação tradicional.

Outro importante aspecto a ser considerado diz respeito à compreensão do pesquisador sobre o fato de, nessa abordagem de pesquisa, as demandas emergentes não serem previsíveis, visto que todas as etapas do estudo são constantemente negociadas entre os parceiros acadêmicos e comunitários. ${ }^{3-4}$ Em outras palavras, que o pesquisador não detém o controle absoluto do processo e, portanto, deve ter muita habilidade para respeitar o movimento da comunidade e considerar as necessidades do CAC, sem perder de vista os pressupostos do rigor da pesquisa qualitativa. ${ }^{6,8,17-18}$ Como a participação no CAC, geralmente é voluntária, cabe ao pesquisador também deve ser flexível com relação à disponibilidade ou não dos membros para participar de algumas ações.

Nesse sentido, o conhecimento sobre coordenação de grupos constitui um importante capital intelectual para o pesquisador, pois auxilia na preservação do espírito de equipe, bem como desenvolve e cultiva o seu melhor potencial. ${ }^{19}$ Outras qualidades também são importantes e devem ser valorizadas na relação entre o pesquisador e o CAC: gerenciamento de conflitos, a desconfiança no processo, a valorização das diversidades culturais e sociais, a manutenção do respeito mútuo e participação efetiva de todos os parceiros..$^{3-4,6,8-9}$

A melhor compreensão dos objetivos da pesquisa e a parceria ao longo do tempo são fatores que contribuirão para o relacionamento de extrema confiança entre os envolvidos no 
Pesquisa participante baseada na comunidade: fundamentos, requisitos e desafios ao pesquisador | 12

processo, bem como para a sustentabilidade desta parceria por período indeterminado. Todavia, o pesquisador deve compreender que os interesses dos membros comunitários podem ser modificados com o passar do tempo, o que tornaria necessário engajar outras pessoas no CAC. ${ }^{3-4}$

Outras dificuldades apontadas na literatura sobre o uso da PPBC foram relacionadas ao prazo determinado para término da pesquisa por parte das agências de fomento e a restrição de tempo dos parceiros comunitários. ${ }^{3,5}$ Nessa abordagem, as pesquisas são construídas a longo prazo, sendo fundamental negociar os problemas prioritários a serem estudados/resolvidos a curto, médio e longo prazo, bem como garantir a continuidade das ações investigativas de interesse da comunidade. Adicionalmente, deve-se prever como atender aos prazos estabelecidos para apresentar relatórios às agências de fomento, pois dificilmente a pesquisa se finda na primeira coleta de dados. Dada a característica cíclica da PA, uma ação leva a outras nem sempre previstas inicialmente. ${ }^{4}$

Para a adequada condução de uma PPBC, estudo ${ }^{5}$ sugere algumas indagações iniciais (Quadro 2):

\begin{tabular}{|c|c|}
\hline QUESTÕES INICIAIS & DETALHES A OBSERVAR \\
\hline O que pesquisar? & Quais serão as questões e o desenho de pesquisa? \\
\hline Como pesquisar? & Como nos envolvemos em parceria? \\
\hline Quem estará na pesquisa? & Como as identidades dos parceiros se \\
& relacionam? \\
\hline
\end{tabular}

Quadro 2 - Questões essenciais e detalhes para o planejamento de uma Pesquisa Participante Baseada na Comunidade

A aproximação dos autores deste texto com a PPBC teve início após a realização de estudos fundamentados na PA, desenvolvidos com a comunidade latina, ${ }^{3}$ no contexto da atenção à saúde de cuidadores de $\operatorname{idosos}^{20} \mathrm{e}$, mais recentemente, por ocasião de uma pesquisa realizada com comunidades urbanas para investigar os riscos do uso abusivo de álcool e outras drogas, que ainda não foi finalizada. No entanto, apesar dessa experiência prévia em envolver os integrantes da pesquisa na definição do objeto de pesquisa, comum na PA, a PPBC trouxe uma 
importante inovação ao incluir o estudo com e em comunidade. Nesse caso, o poder de decisão dos rumos da investigação não está centrado no pesquisador que se abre às novas perspectivas da comunidade sobre a problemática e respeita o que ela considera prioritário e emergencial, ainda que isso possa desafiá-lo nas suas intenções ou percepções do melhor caminho a seguir.

Finalmente, diante da complexidade e diversidade que envolvem o desenvolvimento de estudos orientados pela PPBC, deu-se início a um estudo internacional para validação de um protocolo padrão-ouro com vistas a definir o planejamento e condução de pesquisas utilizando a PPBC. ${ }^{21-24}$ Certamente, esse protocolo será extremamente útil para nortear futuras investigações e pesquisadores iniciantes no uso dessa abordagem.

\section{Considerações finais}

A PPBC é um método inovador para a realização de pesquisas que envolvem comunidades, pois valoriza a participação comunitária em todas as fases do processo investigativo e viabiliza intervenções condizentes com as reais necessidades da população em estudo. A contribuição do presente artigo foi trazer para a discussão os principais conceitos que fundamentam essa abordagem metodológica e os desafios impostos ao pesquisador interessado em adotá-la, além de contribuir para preencher uma lacuna de conhecimento a esse respeito identificada na literatura nacional.

Mesmo que uma comunidade tenha seu empoderamento local e consiga gerenciar os problemas pertinentes à sua realidade, o conhecimento produzido mediante o uso da PPBC é extremamente relevante, pois pode favorecer outras comunidades com características similares e, assim, proporcionar mudanças efetivas e necessárias em contextos semelhantes. Contudo, ao adotar a PPBC como abordagem metodológica, o pesquisador necessita desenvolver algumas competências essenciais, como: a compreensão da missão e dos valores dessa abordagem de pesquisa, conhecer os constructos teóricos-filosóficos e, sobretudo, ser capaz de implementar 
Pesquisa participante baseada na comunidade: fundamentos, requisitos e desafios ao pesquisador I 14

simultaneamente o processo de investigação e a intervenção, por meio de um processo contínuo de aprendizagem compartilhada.

Abordagens metodológicas participativas criam espaços e instrumentos de produção e divulgação de ideias, práticas e alternativas de cuidado que vão ao encontro do princípio da universalidade. Isso permite ir além da distribuição e oferta do cuidado e diminui barreiras de linguagem e comunicação entre profissionais dos serviços, pesquisadores e usuários do sistema de saúde. Nesse sentido, a PPBC auxilia no gerenciamento diário do cuidado por meio da sustentação do diálogo cultural e do fazer compartilhado, desenvolvendo confiança mútua e sustentabilidade ao longo do processo de interação entre pesquisador-comunidade.

Finalmente, os princípios da PPBC favorecem que parceiros acadêmicos e comunitários produzam conhecimento acoplado ao cuidado, sendo altamente adequada ao contexto da saúde pública, uma vez que estimula o processo de gestão participativa e propicia o desenvolvimento e aperfeiçoamento das tecnologias leves e, consequentemente, de transformações psicossocioculturais. Além disso, pode representar uma abordagem efetiva para atender as exigências das agências de fomentos para pesquisas que envolvem a comunidade, no sentido de maximizar a relevância dos resultados sem perder o rigor o metodológico, e uma importante ferramenta para traduzir o conhecimento produzido no âmbito acadêmico em resultados que atendam aos interesses comunitários.

$\mathrm{Na}$ Enfermagem, em particular, considerando que os enfermeiros desempenham papel ativo na promoção e proteção da saúde, sugere-se que a PPBC seja adotada como uma abordagem científica viável para ampliar a compreensão a respeito da complexidade humana e do avanço da enfermagem comunitária. 


\section{Referências}

1. Scochi CGS, Gelbcke FL, Ferreira MA, Lima MADS, Padilha KG, Padovani NA, et al. Nursing doctorates in Brazil: research formation and these production. Rev Latinoam Enferm [Internet]. 2015 jun [acesso em 2018 fev 04];23(3):387-94. Disponível em:

http://www.scielo.br/scielo.php?script=sci_arttext\&pid=S0104-11692015000300387\&lng=en doi:10.1590/0104-1169.0590.2564

2. Costa LS. Innovation in health care services: notes on the limits offield research. Cad Saúde Pública [Internet]. 2016 nov [acesso em 2018 maio 04];32(Supl 2):e00151915. Disponível em: http://www.scielo.br/scielo.php?script=sci_arttext\&pid=S0102-311X2016001402004\&lng=en. doi:10.1590/0102-311X00151915

3. Stacciarini JMR. Pesquisa participante baseada na comunidade: trabalhando com e para latinos rurais no norte da Florida - EUA. Rev Enferm UERJ [Internet]. 2014 nov-dez [acesso em 2018 abr 30];22(6):843-7. Disponível em: https://www.e-publicacoes.uerj.br/index.php/enfermagemuerj/article/view/15779/12284 doi: 10.12957/reuerj.2014.15779

4. Israel BA, Eng E, Schulz AJ, Parker EA, Satcher D. Methods of community based participatory research. San Francisco (CA): Jossey-Bass; 2013.

5. Muhammad M, Wallerstein N, Sussman AL, Avila M, Belone L, Duran B. Reflections on researcher identity and power: the impact of positionality on community based participatory research (CBPR) processes and outcomes. Crit Sociol [Internet]. 2015 nov [acesso em 2018 fev 10];41(7-8):1045-63. Disponível em: https://www.ncbi.nlm.nih.gov/pmc/articles/PMC4943756/ doi: 10.1177/0896920513516025

6. Ward M, Schulz AJ, Israel BA, Rice K, Martenies SE, Markarian E. A conceptual framework for evaluating health equity promotion within community-based participatory research partnerships. Eval Program Plann [Internet]. 2018 out [acesso em 2018 jun 29];30(70):25-34. Disponível em: https://www.sciencedirect.com/science/article/abs/pii/S0149718917303166?via\%3Dihub doi: 10.1016/j.evalprogplan.2018.04.014

7. Freire P. Creating alternative research methods: learning to do it by doing it. New Delhi: Society for Participatory Research in Asia; 1982.

8. Wallerstein N, Giatti LL, Bógus CM, Akerman M, Jacobi PR, Toledo RF, et al. Shared participatory principles and methodologies: perspectives from the USA and Brazil-45 Years after Paulo Freire's "Pedagogy of the oppressed". Societies [Internet]. 2017 abr [acesso em 2018 jan 30];7(2):6. Disponível em: https://www.mdpi.com/2075-4698/7/2/6/htm doi: 10.3390/soc7020006

9. Green LW, George MA, Daniel M, Frankish CJ, Herbert CP, et al. Study of participatory research in health promotion: review and recommendations for the development of participatory research in health promotion in Canada. Vancouver: Royal Society of Canada; 1995. 
Pesquisa participante baseada na comunidade: fundamentos, requisitos e desafios ao pesquisador | 16

10. McElfish PA, Ayers BL, Purvis RS, Long CR, Sinclair K, Esquivel M, et al. Best practices for community-engaged participatory research with Pacific Islander communities in the USA and USAPI: protocol for a scopin greview. BMJ Open [Internet]. 2018 jan [acesso em 2018 jan 21];24:8(1):e019653. Disponível em: https:/www.ncbi.nlm.nih.gov/pmc/articles/PMC5786128/ doi: 10.1136/bmjopen-2017019653

11. Israel BA, Schulz AJ, Parker EA, Becker AB. Review of community-based research: assessing partnership approaches to improve public health. Annu Rev Public Health. 1998;19:173-202.

12. ViswanathanM, Ammerman A, Eng E, Garlehner G, Lohr KN, Griffith D, et al. Community-based participatory research: assessing the evidence. Rockville: Agency for Health Care Research and Quality; 2004.

13. Robertson-James C, Sawyer L, Núñez A, Campoli B, Robertson D, DeVilliers A, et al. Promoting policy development through Community Parcipatory Approaches to health promotion: the Philadelphia ujima experience. Womens Health Issues [Internet]. 2017 out [acesso em 2018 abr 20];17(27 Supl1):S29S37. Disponível em: https://www.whijournal.com/article/S1049-3867(16)30395-4/fulltext doi: http://dx.doi.org/10.1016/j.whi.2017.09.001

14. Furgurson KF, Sandberg JC, Hsu FC, Mora DC, Quandt SA, Arcury TA. Cancer knowledge among mexican immigrant farmworkers in North Carolina. J Immigr Minor Health [Internet]. 2018 jun [acesso em 2018 jun 30];21(3):515-21. Disponível em: https:/www.ncbi.nlm.nih.gov/pubmed/29948650 doi: 10.1007/s10903-018-0771-0

15. MacDonald T, Jackson S, Charles MC, Periel M, Jean-Baptiste MV, Salomon A, et al. The fourth delay and community-driven solutions to reduce maternal mortality in rural Haiti: a community-based action research study. BMC Pregnancy Childbirth [Internet]. 2018 jun [acesso em 2018 jun 29];18(1):254. Disponível em: https://www.ncbi.nlm.nih.gov/pmc/articles/PMC6011389/ doi:10.1186/s12884-018-1881-3

16. Windsor L. Using concept mapping in community-based participatory research: a mixed methods approach. J Mix Methods Res [Internet]. 2013 mar [acesso em 2018 abr 30];7(3):274-93. Disponível em: https://www.ncbi.nlm.nih.gov/pmc/articles/PMC4638322/ doi: 10.1177/1558689813479175

17. Arora PG, Krumholz LS, Guerra T, Leff SS. Measuring community-based participatory research partnerships: the initial development of an assessment instrument. Prog Community Health Partnersh [Internet]. 2015 [acesso em 2018 jun 29];9(4):549-60. Disponível em: https://www.ncbi.nlm.nih.gov/pubmed/26639381 doi:10.1353/cpr.2015.0077

18. Dias S, Gama A. Investigação participativa baseada na comunidade em saúde pública: potencialidades e desafios. Rev Panam Salud Pública [Internet]. 2014 [acesso em 2018 jan 15];35(2):150-4. Disponível em: https://www.scielosp.org/article/rpsp/2014.v35n2/150-154/ 
19. Claudio L, Gilmore J, Roy M, Brenner B. Communicating environmental exposure results and health information in a community-based participatory research study. BMC Public Health [Internet]. 2018 jun [acesso em 2018 jun 30];25;18(1):784. Disponível em: https://www.ncbi.nlm.nih.gov/pmc/articles/PMC6019712/ doi: 10.1186/s12889-018-5721-1

20. Borges CJ. Estratégias de atenção aos cuidadores informais de idosos: pesquisa participante baseada na comunidade [tese]. Goiânia: Universidade Federal de Goiás; 2017.178 p.

21. Lucero J, Wallerstein N, Duran B, Alegria M, Greene-Moton E, Israel B, et al. Development of a mixed methods investigation of process and outcomes of community-based participatory research. J Mix Methods Res [Internet]. 2018 fev [acesso em 2019 jan 21];12(1):55-74. Disponível em: https://www.ncbi.nlm.nih.gov/pmc/articles/PMC5722275/ doi: 10.1177/1558689816633309

22. Oetzel JG, Zhou C, Duran B, Pearson C, Magarati M, Lucero J, et al. Establishing the psychometric properties of constructs in a community-based participatory research conceptual model. J Mix Methods Res [Internet]. 2015 abr [acesso em 2019 jan 18];29(5):e88-e02. Disponível em: https://www.ncbi.nlm.nih.gov/pmc/articles/PMC4819435/ doi:10.4278/ajhp.130731-QUAN-398

23. McElfish PA, Moore R, Laelan M, Ayers BL. Using CBPR to address health disparities with the Marshallese community in Arkansas. Ann Hum Biol [Internet]. 2018 maio [acesso em 2019 jan 20];45(3):264-71. Disponível em: https://www.ncbi.nlm.nih.gov/pubmed/29877159 doi: 10.1080/03014460.2018.1461927

24. Belone L, Lucero JE, Duran B, Tafoya G, Baker EA, Chan D, et al. Community-based participatory research conceptual model: community partner consultation and face validity. Qual Health Res [Internet]. 2014 [acesso em 2019 jan 20];26(1):117-35. Disponível em: https://www.ncbi.nlm.nih.gov/pmc/articles/PMC4839192/ doi: 10.1177/1049732314557084

\section{Autor correspondente}

Nome: Cristiane José Borges

E-mail: cristianejose@yahoo.com.br

Endereço: Curso de Enfermagem - Universidade Federal de Goiás - Regional Jataí. BR 364, KM 195, n³800 Câmpus Cidade Universitária - CEP: -Jataí-Goiás.

CEP: 75801-615

\section{Contribuições de Autoria}

\section{1 - Cristiane José Borges}

Concepção e planejamento do projeto de pesquisa, obtenção, análise, interpretação dos dados e redação.

\section{2 - Denize Bouttelet Munari}


Pesquisa participante baseada na comunidade: fundamentos, requisitos e desafios ao pesquisador I 18

Contribuições: Concepção e Planejamento do projeto de pesquisa, redação e revisão crítica.

3 - Veridiana Carvalho Bianco

Obtenção e análise de dados.

4 - Paula Cândida da Silva Dias

Obtenção e análise de dados.

5 - Marcelo Medeiros

Revisão crítica.

6 - Jeanne-Marie Rodrigues Stacciarini

Planejamento do projeto de pesquisa e revisão crítica.

\section{Como citar este artigo}

Borges CJ, Munari DB, Bianco VC, Dias PCS, Medeiros M, Stacciarini JMR. Pesquisa participante baseada na comunidade: fundamentos, requisitos e desafios ao pesquisador. Rev. Enferm. UFSM. 2019 [Acesso em: Anos Mês Dia];vol e48: P1-P18. DOI:https://doi.org/10.5902/2179769232536 